\title{
The Enabling Role of Social Media for Chinese Immigrants' Health: A Pilot Study
}

\section{Erting Sa and Ricky Leung}

University at Albany, SUNY, USA

*Corresponding author: Ricky Leung, One University PI., Rm. 181, School of Public Health, Rensselaer NY, 12144, USA, Tel: 518-402-6512; E-mail: rleung@albany.edu

Received date: June 04, 2016; Accepted date: July 21, 2016; Published date: July 29, 2016

Copyright: ( 2016 Sa E, et al. This is an open-access article distributed under the terms of the Creative Commons Attribution License, which permits unrestricted use, distribution, and reproduction in any medium, provided the original author and source are credited

\begin{abstract}
Previous research has shown that obtaining health information-including where to locate a doctor and seek appropriate treatment-is a major challenge for Chinese and other Asian immigrants to adapt to the US. Although social media could provide Chinese immigrants with health information and other benefits, the new technology's value is still uncertain for potential users. This pilot study utilizes a qualitative research design to better understand the ambivalent attitudes of Chinese immigrants towards social media. We found that Chinese immigrants still perceived social media as having a number of shortcomings. Yet, if used properly, social media could be very useful for these immigrants to seek and share health information with their peers. Based on interview results, we identified several opportunities for developing social media tools, and directions for further research.
\end{abstract}

Keywords: Social media; Chinese immigrants; Adaptation; Informal care

\section{Introduction}

Chinese immigrants are the third-largest immigrant group in the United States (US) in 2013 [1]. Chinese immigrants tend to focus on building social ties within their own ethnic group, so that they often have weak social ties with the general population [2]. This has sometimes led to challenges for Chinese immigrants to adapt to the new social environment in the US. Previous research has shown that obtaining health information-including where to locate a doctor and seek appropriate treatment-is one of these major challenges for Chinese and other Asian immigrants [3].

Tackling this challenge is not easy. Most importantly, Chinese immigrants constitute a very diverse group with different health and social needs. Older Chinese immigrants often suffer from depression, arthritis, back or neck problems that are common to old people [4], and they are mostly interested in learning how to adopt and support healthy living [5]. Younger Chinese immigrants have different needs, depending on immigration and job status. For example, young Chinese immigrants who worked in restaurants often had poor insurance coverage. They were particularly concerned with access to critical health care [6].

In the US, social media have become very popular. According to Duggan [7], Facebook has captured as many as $62 \%$ of all American adults. Other social media platforms have also enrolled a very large number of users, including Pinterest, Instagram, Linkedin or discussion forums such as Reddit and Digg. Many people have now used social media to get health information, such as by reading articles online [8], and connecting with a hospital [9]. Can this new technology help Chinese immigrants obtain health information and address other health needs? Do they trust social media for getting health information and what are the perceived benefits of this new technology?
Leung and Li [3] have identified several benefits of social media usage among Asian immigrants. Maintaining relationships with family members in their original home is a critical benefit, because doing so helped Chinese immigrants gain emotional support and reduce depressive symptoms. Social media also enable Chinese and other Asian immigrants to reach health care providers through communitybased and religious activities. Nonetheless, Leung and Li's survey research had limited data and was inconclusive. They found that some Asian immigrants viewed social media as the hotbed of rumors, mistaken information, and ultimately new forms of anxiety and stress. According to their research, using social media too much can impact an immigrant's task performance as well.

In this context, this pilot study aims to clarify the motivations of social media usage among Chinese immigrants in the US. We will examine whether or not and how Chinese immigrants use social media to address health needs with a qualitative research design. This exploratory research will allow us to build on previous research and pave the way for a future study.

\section{Methods}

\section{Participants}

We recruited 10 Chinese immigrants (including parents, children, and spouses of the primary immigrant) who resided in Boston, MA to participate in the study. In the US, the number of Chinese immigrants in Boston-Cambridge-Newton, MA-NH area ranked the fourth highest concentration of Chinese immigrants followed by greater New York City, San Francisco and Los Angeles metropolitan areas in 2013 [1]. Choosing this research site is thus advantageous for planning a followup study. A Chinese community center in Boston's Chinatown area assisted us in recruiting participants. Six of these immigrants were US citizens, and four were "green card holders", as of April 2016. This study received ethical approval from the Institutional Review Board from the University at Albany. Table 1 summarizes selected demographic characteristics of our participants. 


\begin{tabular}{|c|c|}
\hline Gender & \\
\hline Male & 1 \\
\hline Female & 9 \\
\hline Age range & $20-50$ years \\
\hline (Mean age/Std. Deviation) (in years) & $\begin{array}{ll}(31 & \text { years } / 12 \\
\text { years }) & \end{array}$ \\
\hline Years living in the US & $4-25$ years \\
\hline (Mean duration/Std. Deviation) & $\begin{array}{ll}(11 & \text { years } / 7 \\
\text { years }) & \end{array}$ \\
\hline \multicolumn{2}{|l|}{ Educational level } \\
\hline Bachelor & 4 \\
\hline Master & 5 \\
\hline Ph. D. & 1 \\
\hline
\end{tabular}

Table 1: Characteristics of the study sample $(n=10)$.

\section{Data collection and analysis}

A semi-structured interview guide with open-ended questions in both English and Chinese version was used to encourage participants to speak freely about the topics. Participants were required to read and sign the informed consent form before the interview started. Background information, such as age, gender, educational level, etc., was asked. Then open-ended questions such as "What specific social media do you use in daily life?" "How do you use social media?" "How do you think about social media in terms of addressing your health needs?" All interviews were audio-recorded with the participant's consent. The interviewer also wrote additional notes during the interview. Two researchers transcribed interview data in their entirety separately, and then compared the transcripts for coding before the analysis. Following the coding procedures of Emerson et al. [10], we collapsed and grouped codes into categories and themes. We organize results below around these themes.

\section{Results}

\section{Why social media?}

Useful information is available on social media platforms. One participant had been under a high level of work-related stress. She expressed a need to be "out of the work zone" and wanted to share good times with loved one and family members. Social media could provide useful inspirations, and this Chinese immigrant could find "things to do" that were in line with her cultural values. This was very important because she-as were almost all our participants-thought that Chinese immigrants were sometimes being marginalized as a group of "different" people in the US. Social media could provide some cultural support.

Our participants were well-educated and they had relatively stable jobs. As such, they all had good health insurance coverage and a high level of "Internet literacy". While it was not difficult to locate a doctor from the Internet, our participants would use social media to find out doctors' quality and efficiency.
"I am concerned about how to make a medical appointment more efficiently and how to see a doctor efficiently as well. My past experience often made me upset, because I always have to wait for $1 \mathrm{~h}$ or more to see my doctor and the meeting with the doctor only takes up 10 to 20 min".

At the same time, social media could work as a motivational device. Some participants were aware of their unhealthy lifestyle were eager to keep up with a healthy lifestyle, which could involve regular exercises. As one confessed, "It is so difficult for me to keep a healthy lifestyle even if I know what is good for me." But some also highlighted they use social media websites to motivate and regulate their daily exercises. By doing that, not only do they get accustomed to a healthy lifestyle, but also obtain satisfaction from social relationships. As one said,

"I am in an exercise group on WeChat. Every day we will post our "combat gains" to compete with each other, such as how many miles I run today or how many steps I walk today. And most of us don't want to be the one with fewer numbers. Sometimes, we will also work out together. I think this is a meaningful group for me, as I keep fit and make friends."

Interestingly, using social media did not have to be "self-centred". Although most of our participants did not suffer from a chronic illness, nine out of ten said that they had one or more family member(s) or friend(s) who was diagnosed with a chronic illness. Five participants said that they had provided informal care to the family member(s) or friend(s). Based on this caregiving experience, these participants had identified several challenges regarding the use of health services among Chinese immigrants with a chronic illness condition. These challenges included language barriers, health literacy, media literacy, and cultural values. Social media could be utilized to help their family member(s) and/or friend(s) to better prepare for handling these challenges. For example, older family members might have limited English proficiency to communicate with doctors and understand medication instructions. They also did not know how to ask questions properly, and sometimes develop fears of being stigmatized. Social media might be a useful source of information. Our participants could share their experiences with friends and family members who needed help.

Yet, according to our participants, not all immigrants could utilize social media equally well. First and foremost, it could be difficult to extract essential information from social media, and acting on this information might be challenging for some Chinese immigrants. As one participant of ours complained:

"It is always time-consuming to read many long articles to filter out the key messages, and it is a burden for a patient to seek health information as well, so if there is a function that the social media can automatically summarize the key points of the health related articles, that would be great!"

That is, our participants wanted to receive customized contents from social media platforms that were built for Chinese immigrants specifically. They were also interested in new functions and new social media tools, such as a mobile application or "app". These apps would be much desired if they could provide appointment reminders, tutorials for medication adherence and health services in the US, and other educational materials, according to our participants. One of them explained the importance of these new tools this way:

"Many of the Chinese immigrants actually don't know the availability of related resources, so if social media can post public 
announcements or advertisements to inform the patients, that will be helpful."

\section{Still skeptical, but increasingly acceptable}

As the discussions above suggest, Chinese immigrants might still be skeptical about the utility of social media in addressing health needs. But there was increasing acceptance: Out of ten participants in our sample, seven of them generally trusted the contents they received from social media; the other three participants had some negative concerns. One said:

"Anyone can post on these social media, and there are so many misconceptions and misleading information online."

With regard to health information, interestingly, nine participants believed that patients could benefit from social media if they used them properly. Our participants cited such things as "easy-to-use", "convenient", "helpful" and "informative" as the advantages of social media for patients. As one participant put it:

"There is good information that patients can learn from on the social media and they can get involved in, maybe, a support group to exchange treatment experience."

Easy-to-use and convenient aside, there was another specific reason behind Chinese immigrants' increasing acceptance towards social media for getting health-related information. That is, they could use the technology to connect with friends and family members of their old home to be informed of health-related matters. Explained by one of our participants:

"Nowadays, any explosive or popular [health-related] news will be easily shared and reposted by social media users, so it is really easy to get information about what is going on around the world. For me, if I want to know what is happening in China, the easiest way is to follow a Chinese news account in my Weibo (Chinese version Twitter)."

Weibo and other Chinese social media platforms also allowed Chinese immigrants to obtain health-related information in Chinese. Another two participant of ours said:

"I actually subscribe to some health related information in my WeChat (one of the most popular and multi-functional communication apps in China), and I like to read those articles about how to keep [myself] healthy."

"I am really into some [Chinese form of] Instagram accounts that provide healthy food recipe with really appetite-alluring pictures."

While these Chinese immigrants were interested in Chinese health contents, they could learn English from social media as well. Two participants specifically mentioned that they used social media to learn English, since they were not born in the US and they wanted to adapt to the new environment as soon as possible. Other participants mentioned watching YouTube videos to learn other things, such as cooking, doing make-ups, and putting contact lenses in the Western style.

Among our ten participants, half (5) of them indicated that they preferred using US-based social media than the Chinese ones, simply because they were now in the US. Two of them responded to our interview question in a very similar way:

"I use US social media more often than the Chinese social media, because [this is] where I live, people around me use the US social media more and I need to use then to keep myself updated."
"I use US social media more than the Chinese ones, mainly because I am here in the US. I need to be part of the society and I don't want to be isolated. I want to adapt to the new environment better."

Those who preferred Chinese social media than the US ones mentioned things related to "nativity", "language" and "culture" as the main reasons for their preference:

"It's more convenient for me because it's [Chinese is] my native language and most of my friends are Chinese and we are more connected to the Chinese social media. It's easy for us to share health related articles, news or tips with each other on the shared social media usage."

"I still prefer using Chinese social media as I have been using them for decades, and most of my family members and good friends are on these social media. I use US social media only for work-related purposes."

The other participants were neutral between the US and Chinese social media. These participants believed: "it really depends" [on what purposes to use what type of social media].

\section{Using social media everyday}

Despite skepticism, most of our participants used social media on a daily basis. All the participants indicated that they would definitely check their social media at least once a day. However, use frequency seemed to correlate with educational level negatively, but only slightly. Generally, college students reported using social media more frequently than their higher educated counterparts, as one said:

"I like to check my social media sites every time when a message reminder pops out in my iPhone. I want to know what happened to my friends and I like to post on my social media to keep everyone updated about my cool news."

Our participants with a master or doctoral degree were more restrained in spending social media time. One of them put it this way:

"I tend to check my social media every morning on my way to work on the train to see what's going on and every night before I go to bed to see what's new [and that's all]."

During the interviews, our participants actually showed that they had very good knowledge about a variety of social media platforms. To connect with Chinese family members and friends, Chinese social media platforms were typically preferred to US ones. These platforms included WeChat (mobile text and voice messaging apps), Weibo (Chinese version Twitter), QQ (one of the earliest text messaging tool in China), Zhihu (Online forums) and Douban (Online forums). And some participants believed that different platforms were more suitable for connecting with certain friends or family members. For example, one participant said:

"I use WeChat more for connecting with my parents and grandparents."

Our participants did not seem to have any specific preference for using US social media to connect with their non-Chinese social ties. Yet, they knew of many different US-based social media platforms, including Facebook, Twitter, Instagram, YouTube, Snapchat, Linkedin, WhatsApp, Skype, and GroupMe. Facebook and YouTube were the most frequently mentioned and preferred social media in our participants' daily life. 


\section{Discussion}

This pilot study has led to a number of interesting findings that can become the basis for a larger study. We found interesting results of how Chinese immigrants used social media and how they use it for health related purpose. Although the use of social media to address health needs seems to be a non-mainstream method for them, it has a big potential to be exploited to benefit users by providing customized health information. In short, Chinese immigrants had an ambivalent attitude towards social media for addressing health information and other needs. Based on our participants, for instance, social media sometimes had too much information, making it difficult to digest. Besides, some contents might not be of interest for Chinese immigrants. This finding can become a profitable business opportunity for entrepreneurs. They can develop new social media platforms (or related tools such as mobile apps) that are customized to Chinese users. Contents in these customized platforms should be condensed, not just easy-to-use but also easy-to-understand.

According to our participants, it could be very difficult for Chinese immigrants to distinguish between accurate and inaccurate information. In this regard, it will be very useful for future researchers to develop evaluative metrics to help Chinese and other immigrants assess social media contents-even the entire social media platformmore systematically. With more developed evaluative metrics, Chinese immigrants would be more confident in seeking health information from social media, and they could also share their knowledge with peers more comfortably.

Finally, it is noteworthy that Chinese immigrants could benefit from social media indirectly as well as directly. That is, they need not use the information to improve health themselves, but they could learn useful things for friends and family members (such as those who had chronic diseases), and perhaps provide better informal care for friends and family members in need. Also, while social media enabled our immigrant participants to gain emotional support, they offered a channel for these immigrants to "give back" to peers and even the immigrant community. Giving help can be a very satisfying experience by itself. This finding has significant implications for informal care research and practice. In future research, it is worth exploring this philanthropic dimension of social media among Chinese and other immigrants. Possibly, an increased capacity to help significant others is why immigrants-and other population groups-would enjoy using social media for obtaining health information.

\section{Limitation}

Since this is a pilot with a small sample size, our findings may not be generalizable to all Chinese immigrants in the US. Moreover, we could not recruit participants of more diversified backgrounds, so our findings might have missed important aspects regarding health information and behaviors among immigrants. Yet, our findings can serve as a useful basis to motivate further studies on this interesting and important topic. We are particularly interested in collecting more data from a large-scale study, because these data are necessary to verify the pilot findings reported in this paper.

\section{Conclusion}

This pilot study enables us to understand how Chinese immigrants use social media in the US at present. The results provide us with the reasons, motivations, preferences and health-related purpose of the usage of social media among them. More importantly, we have seen both opportunities and challenges opportunities of using social media to address their health needs in the future, as we find out that they have obtained health-related information from social media, they have treated social media as an access to gain social support, and they indeed have the demand and expectation to obtain more health benefits from social media. Nonetheless, there are many intangible difficulties that researchers and developers may need to address in both conducting more relevant research and developing better social media systems.

\section{References}

1. Hooper K, Batalova J (2015) Chinese immigrants in the United States. Migration Policy Institute.

2. Romero I, Yu Z (2015) Analyzing the influence of social capital on selfemployment: A study of Chinese immigrants. Annals of Regional Science 54: 877-899.

3. Leung R, Li J (2015) Using social media to address Asian immigrants' metal health needs: A systematic literature review. Journal of Nature and Science 1: 1-5.

4. Wu B, Chi I, Plassman BL, Guo M (2010) Depressive symptoms and health problems among Chinese immigrant elders in the US and Chinese elders in China. Aging Ment Health 14: 695-704.

5. Liu Z, Speed S, Beaver K (2012) Perceptions and attitudes towards exercise among Chinese elders - the implications of culturally based selfmanagement strategies for effective health-related help seeking and person-centered care. Health Expectations, 18: 262-272.

6. Gao H, Dutta M, Okoror T (2016). Listening to Chinese Immigrant Restaurant Workers in the Midwest: Application of the Culture-Centered Approach (CCA) to Explore Perceptions of Health and Health Care. Health Communication 31: 727-737.

7. Duggan M (2015) Mobile messaging and social media 2015. PEW Research Center.

8. Pálsdóttir A (2014) Preferences in the use of social media for seeking and communicating health and lifestyle information. Information Research 19: 1-16.

9. Leung R, Pasupathy KS (2011) The economics of social computing: some preliminary findings on healthcare organizations. Journal of computational Science 2: 253-261.

10. Emerson RM, Fretz RI, Shaw LL (2011) Writing ethnographic fieldnotes. University of Chicago Press. 